Revista de Investigación Educativa 5

julio-diciembre 2007 | ISSN 1870-5308 | Xalapa, Veracruz

Instituto de Investigaciones en Educación | Universidad Veracruzana

\title{
¿Una psicología de la educación?
}

Jean-Marie Besse

Albertina Huerta Galván

Traducción

En este artículo se discute la pertinencia de los distintos esfuerzos por hacer intervenir a las disciplinas psicológicas en el ámbito de la educación, de lo cual han derivado denominaciones como "psicopedagogía", "ciencia de la educación", "investigación pedagógica", "experimentación educativa". Se cuestiona esta intención de establecer un acercamiento científico de la infancia y de la educación. Para ello, se analizan y confrontan las reflexiones de Decroly donde se cruzan psicología y educación, ciencia y pedagogía, la obra "psicopedagógica" de A. Binet y algunas intervenciones "pedagógicas" de J. Piaget.

Palabras clave: Psicología educativa, psicopedagogía, Decroly, Piaget, Binet.

Para citar este artículo:

Besse, J. M. (2007, julio-diciembre). ¿Una psicología de la educación?. CPU-e, Revista de Investigación Educativa, 5. Recuperado el [fecha de consulta], de http://www.uv.mx/cpue/num5/ inves/besse_psicologia_educacion.html 


\section{¿Una psicología de la educación?}

$\mathrm{D}$ esde los orígenes mismos de la psicología - la elaboración de una psicología autónoma-, la intención de intervenir en el terreno educativo obligó a los primeros investigadores a definir sus objetivos, sus metodologías, sus referentes teóricos, las modalidades de sus aportaciones eventuales a la pedagogía. "Psicopedagogía", "ciencia de la educación”, "investigación pedagógica”, "experimentación educativa"... tales formulaciones pretendieron describir entonces esos intentos y las ambiciones proclamadas.

Ahora bien, estas terminologías se entrecruzan, coinciden con otras, remiten a diversos significados. ¿Qué hay de su pertinencia y de su interés? Para retomar el estudio de la práctica y de las reflexiones más teóricas de Decroly no dejemos de advertir que su obra se ha situado precisamente en esa encrucijada de las investigaciones, entre psicología y educación, entre ciencia y pedagogía.

También podría ser útil, por lo menos, cuestionar esta intención de establecer un acercamiento científico de la infancia y de la educación, separar los motivos de las ambigüedades terminológicas, las oposiciones a las cuales pueden remitir, las posturas que guardan: la relectura de la obra "psicopedagógica" de A. Binet, algunas intervenciones "pedagógicas" de J. Piaget, confrontadas a las posiciones propias de O. Decroly, con la perspectiva de casi un siglo de investigaciones en este campo, ¿no sería susceptible de llevar a una reflexión mejor informada, incluso a prácticas e intervenciones más operantes?

\section{Ambigüedades de la noción de "psicopedagogía"}

El propósito de una psicología de la educación supone un campo de estudio definido y circunscrito de manera operatoria. Sin embargo, ese proyecto no deja de encontrar numerosas dificultades y resistencias, más o menos ocultas pero con frecuencia efectivas, y que muestran, en parte por cierto, que ese campo de estudio es muy disputado, ya que constituye una zona fronteriza e implica así operaciones de "precedencia" sobre un territorio, y por tanto, confrontaciones entre esos acercamientos a las finalidades antagonistas que son, por un lado, la intención educativa y la investigación y la intervención psicológica. 
La apuesta por una definición esclarecedora podría permitir repensar la identidad y las funciones del psicólogo investigador en educación, a reserva de conformarse con esas denominaciones comunes, cómodas sin duda alguna, pero apresuradas, como son las de "psicopedagogía" o de "investigador en ciencias de la educación".

El psicólogo de la educación pide prestado su objeto a la educación, y sus métodos y procedimientos a la psicología. También corre el riesgo de que los pedagogos lo consideren sospechoso de robo de objeto y de imperialismo "psicologizante", mientras que los psicólogos no verán en él más que a un especialista en "psicología aplicada". Eso no impide que, "frente a un psicólogo que no se define de otro modo más que por su título, se desencadenen las expectativas más diversas (entre los profesores): hay quien espera la junta que va a resolver todo, quien espera una investigación sobre la personalidad inmadura de los alumnos, quien pide una bibliografía para poner al día sus competencias pedagógicas, etc." (Selvini, I980: 62) La ambigüedad así manifiesta ¿no es la consecuencia lógica de un papel paradójicamente presentado como susceptible de conducir al conocimiento óptimo del ser humano?

Sin embargo, la psicología de la educación apunta sobre todo al estudio de las conductas presentadas en el ámbito escolar, no es esa "disciplina fundamental que se dedica a la explicación de las conductas educativas en situaciones institucionales" (Gilly, I980: 20), pero se interesa igualmente en transformar las actitudes "equivocadas": en este sentido, se pregunta sobre los procesos de cambio "bajo sus diversas dimensiones: representaciones y valores colectivos, rol $^{1}$ y representaciones de roles, relaciones profesionales y pedagógicas, orientación y formación..." (Maisonneuve, I975: 469) ¿No se puede entonces englobar su tarea bajo esta formulación general: "el psicólogo de la educación es aquel que toma por objeto específico el estudio científico de los procesos interindividuales gracias a los cuales se operan cambios individuales"? (Gilly, r98г: 9).

La psicología de la educación es susceptible de abordar su objeto con préstamos de metodologías heterogéneas (clínica y experimental), pero no se reduce a una psicopedagogía definida como "una pedagogía científicamente basada en la psicología infantil” (Piéron, I957: 292) ni a una técnica de cambio social,

I. Utilizaremos el término rol en lugar de papel por ser su uso más común en el campo de la psicología, aunque para otras disciplinas se prefiera emplear el término papel o, en algunos casos, función. [N. de la T.] 
ni a una terapéutica instituida en el ámbito escolar. No obstante, son todas estas imágenes a la vez las que la constituyen en las representaciones habituales de los maestros.

El investigador en psicología de la educación se presenta así bajo muchos rostros: el innovador, el observador objetivo, el facilitador de las relaciones, el especialista en niños...

Sin duda este asunto no es tan reciente. En un número especial del Bulletin de Psychologie consagrado a Psychologie et Éducation (I967, XX (257), IO-I5), M. Debesse estudiaba de manera profunda la cuestión de las relaciones entre "pedagogos y psicólogos", y señalaba los diversos roles y representaciones que de la psicología se forman los maestros.

No pretenderemos resolver aquí las preguntas señaladas anteriormente: la psicología en el campo escolar oscila, tanto en su práctica como en su teorización, de acuerdo con factores que no consideran, ni mucho menos, la sola voluntad de los psicólogos. Pero querríamos intentar mostrar cuáles reflexiones nos inspiran nuestras actividades de investigación psicológica en el ámbito educacional, confrontadas con una relectura de la experiencia de algunos psicólogos de reconocida reputación y cuyo punto en común podría ser el haber encontrado personalmente esas mismas dificultades.

Si la psicología de la educación sucede hoy a las ambiciones de una "psicopedagogía científica”, ¿no es más que el efecto de un cambio de terminología o es necesario ver en esto una modificación notable de las problemáticas?

\section{I.I La "investigación pedagógica"}

Puede hablarse de "investigación pedagógica" a propósito de proyectos particularmente diferentes: desde las tentativas de Jules Celma (I97I: I37) que busca "rebasar la no-directividad", hasta los ensayos oficiales de renovación de la didáctica de tal o cual disciplina de enseñanza, pasando por reformas de estructura, la expresión genérica sigue siendo la misma. Así pues, el psicólogo de la educación puede ser requerido para una operación de seguimiento o de control de esas diversas modalidades de "investigaciones".

Sin embargo, el estudio de los modelos relacionales registrados entre el psicólogo y los pedagogos (en cualquier nivel que se sitúen de la jerarquía, entre el practicante y el responsable administrativo o político) revela los diferentes roles asignados a la psicología según estas situaciones. 
Entonces, la educación demanda a la psicología fortalecerla en su función de integración social y en su actividad cotidiana, así como ayudarla a evolucionar hacia un mejoramiento de sus prácticas, tomando en cuenta la "realidad psíquica" del niño. Normalmente se pueden deducir dos tipos de preocupación entre los pedagogos frente a la "investigación pedagógica":

- la investigación orientada al mejoramiento de los conocimientos sobre el niño en situación escolar.

- la investigación refleja sobre todo un movimiento de adaptación de la educación a la evolución de las preocupaciones actuales.

El primer modelo requerirá del psicólogo para observar de manera "científica" lo que pasa en el campo escolar; publicará los resultados de sus investigaciones, lo que lo llevará a prever, dado el caso, las modalidades de aplicación de las conclusiones obtenidas al término de sus investigaciones. ${ }^{2}$ También puede concebir una relación estricta de dependencia entre la psicología infantil y la pedagogía: el ejemplo de una cierta acepción de la "psicopedagogía", después del movimiento de la Educación Nueva, ilustra la imposición de una situación semejante.

El segundo modelo invita a un modo muy diferente de intervención del psicólogo, porque se sitúa ante todo en una perspectiva de cambio inmediato, ya sea un cambio individual (un profesor decide su práctica solo) o colectivo (una investigación que se apoya en un equipo, o un grupo de instituciones), y ya sea que el cambio se realice antes de haber llamado al psicólogo o que siga su propio criterio, o incluso que la evolución se realice en colaboración. Así pues, el psicólogo, según los casos, habrá de "seguir" (¡en todos los sentidos de la palabra!) las innovaciones para poner sus competencias y sus técnicas al servicio de la comprensión de los fenómenos psicológicos implicados por la "investigación” o por la evaluación, cualitativa o cuantitativa, de los resultados, escolares o de actitudes, o del acompañamiento del cambio (regulación de grupos de innovación)...

Esta tipología no coincide más que parcialmente con la que proponía $\mathrm{R}$. Buyse (I935: 465), cuando distinguía la experimentación pedagógica de la pedagogía experiencial: el psicólogo debe verificar frecuentemente al lado de los pedagogos que le piden intervenir a cuál de estas imágenes remite su acción y cuál es exactamente el estatus que le otorga a la psicología; las ambigüedades son

2. El libro de L. Legrand, Psychologie appliquée à l'éducation intellectuelle (I96I), Delachaux y Niestlé, es una ilustración de esta aplicación de saberes psicológicos a la educación, aplicación emprendida aquí por un pedagogo. 
numerosas, dependiendo de si ellos creen que deben tratar con un especialista en investigación fundamental -la mayoría de las veces un universitario-, o con un psicosociólogo "animador" de un equipo multidisciplinario o incluso con un "evaluador" antes de dar a conocer los mínimos efectos de la investigación sobre los aprendizajes de los alumnos...

Entre la investigación fundamental, "para saber", y la innovación-investigación, "para cambiar la escuela", se perfila a veces un modelo intermedio, el de una investigación-formación, una vez admitido el postulado de que la educación, la de los alumnos como la de los maestros, es permanente, y por lo tanto, que el movimiento mismo de una adaptación constante de la pedagogía a los alumnos actuales es indispensable: la investigación es entonces la obra de un equipo en el cual el psicólogo conserva una especificidad de exterioridad a la acción pedagógica propiamente dicha, pero acepta su implicación en el futuro de la dinámica colectiva. Es evidente que aquí las modalidades de evaluación clásica experimentan una gran alteración. ${ }^{3}$

El psicólogo que acompaña este tipo de experiencias se va a cuestionar sobre los instrumentos y métodos que lo califican habitualmente en las representaciones de los maestros; constatará rápidamente que su formación y sus preocupaciones metodológicas lo sitúan en uno u otro de los modelos anteriormente descritos: así, cuanto más apegada sea su intervención a las modalidades experimentales más tendrá que contar con las reticencias nacidas de una implicación percibida como insuficiente, de la pesadez y de la lentitud de los dispositivos instalados, de los resultados poco demostrativos, difícilmente legibles y "explotables". Pero la dimensión clínica tampoco está exenta de resistencias, debido a la implicación a la que remite.

Porque la psicología no es objeto de una representación unívoca, más bien refracta la atracción entre el mejor conocimiento del hombre que propone el psicólogo y la inquietud concomitante surgida de los posibles efectos sobre el control de los individuos que una ciencia tan eficaz no podría dejar de suscitar. Entre el temor de un dominio de las técnicas psicológicas -los tests, la evaluación de los alumnos... y de los maestros...-, la esperanza de un dominio de los conocimientos acerca del desarrollo cognitivo que permitan establecer un aprendizaje rentable, ${ }^{4}$ la justificación de la liberación de las fuerzas pulsionales,

3. Un ejemplo de estas tentativas puede ser leído en el texto de L. Allal, J. Cardinet y Ph. Perrenoud (I979) L'évaluation formative dans un enseignement différencié.

4. Pensemos aquí en la utilización de los trabajos piagetianos para sostener los cambios de las 
en una concepción libertaria, con una cierta lectura psicoanalítica, ${ }^{5}$ y el llamado a ciertas formas de "no-directividad" racionalizado por un discurso sobre la expresión infantil "auténtica", las representaciones de la psicología referidas carecen de algo de unidad.

Así, la investigación pedagógica exige intervenciones psicológicas variadas, al azar de las ofertas y de los conocimientos, o la influencia constante de las diversas corrientes: no ofrece la apariencia de una estrategia racionalmente ordenada para que las perspectivas incluyan una distribución organizada de las tareas entre la o las psicologías y la educación.

\section{I.2 La institución escolar, la psicología y la investigación pedagógica}

Sin duda siempre ha existido una u otra forma de "investigación pedagógica" desde el momento en que la intención misma de instruir se manifestó. Para limitarnos a los periodos recientes relativos a la situación francesa, recordemos solamente que la educación tuvo su época de confianza en un progreso asegurado por las conquistas científicas: la pedagogía experimental de principios de este siglo es testigo de tales ambiciones. Luego vino el tiempo de las actividades de investigación instituidas, de la lenta evolución del público de esas investigaciones, el tiempo de la duda. ${ }^{6}$ Sobrevino entonces el momento del replanteamiento más general y para algunos de proclamar "el Año I de la investigación pedagógica", en I968 (Legrand, I968).

De esta manera, numerosas innovaciones pedagógicas verán la luz, pero suscitarán rápidamente la desconfianza oficial: una circular (número 7I-I I del 8 de enero de I97I, publicada en el Boletín Oficial del Ministerio de la Educación Nacional, BOEN, el I4 de enero del 7I) alertó sobre "las iniciativas sin duda generosas pero que, llevadas sin consejos ni control, corren el riesgo de acabar en fracasos cuya consecuencia comprometería los métodos activos que queremos promover". Paralelamente a esta "investigación espontánea" se desarrollará la

“matemáticas modernas", por ejemplo.

5. Cf. nuestro análisis sobre este tema en La pédagogie au XXème siècle, obra colectiva dirigida por Avanzini, Toulouse: Privat.

6. Prueba de esta posición es el artículo de S. Roller (I968), L'aspect institutionnalisé de la recherche en Europe. Cf. también G. Avanzini, I975: 3 I9. 
investigación sobre el programa nacional, confiado a un Instituto Nacional de Investigación Pedagógica, que se esforzará, bajo la dirección de L. Legrand, en promover "innovaciones controladas a gran escala".

No se debería olvidar aquí el papel encomendado a los psicólogos escolares: "El psicólogo escolar debe (...) contribuir a la constitución de una verdadera psicopedagogía. En la encrucijada del saber científico y de la acción educativa, el psicólogo es un elemento irremplazable de esta búsqueda, de la cual su imagen misma es el diálogo cotidiano con el pedagogo.

El psicólogo escolar coopera en el seno mismo del equipo educativo con la reflexión y la elaboración continua de las perspectivas y de los medios de la pedagogía (objetivos, métodos, técnicas). Participa en los trabajos emprendidos en el marco de las circunscripciones de inspección o en el de otros organismos de investigación pedagógica para el progreso teórico y práctico de la actividad educativa".

Las secciones de Ciencias de la Educación de las universidades participan en la animación de la investigación básica; con frecuencia cuentan con laboratorios llamados de pedagogía experimental, de psicología pedagógica, de psicopedagogía, de psicología de la educación...

Esas diversas intervenciones o posibilidades institucionales han tenido diversos logros ${ }^{9}$ y participan de estrategias diferentes. Sin embargo, tienen en común la amplia "utilización" de los psicólogos que ellas requieren para una función de "control": la psicología es percibida -y descrita- como uno de los medios privilegiados aptos para permitir una evolución ordenada de la pedagogía, sin excesos, casi sin incertidumbre...

Desde este punto de vista, dos imágenes nos parecen demostrativas. La psicología diferencial ha provisto por mucho tiempo al modelo de una intervención "edificada" en el terreno de la educación: el empleo de los tests o la referencia a los tests como instrumento "serio", susceptible de servir de guía durante la elaboración de pruebas de evaluación pedagógica. Actualmente la docimología ${ }^{10}$

7. Según la distinción formulada por este autor en Pour une politique démocratique de l'éducation (I977). También se podrá remitir, del mismo autor, a L'évolution des CES expérimentaux (I976).

8. Extracto del informe de un comité paritario reunido después de I968, citado en Andrey y Le Men, I974: 56. Igualmente se podrá remitir a Marc, I977: 237.

9. Hace poco fue lanzado un "Appel pour la recherche en éducation", con el cual se denuncia el estado actual de "subdesarrollo", Le Monde, 28.I.82.

Io. Término propuesto por el psicólogo Henri Piéron, para denominar el estudio científico de las pruebas de exámenes y en particular de los diversos sistemas para clasificar, y de los factores subjetivos que actúan sobre los examinadores. Merani, Alberto. (1982) Diccionario de pedagogía. 
clásica cede el lugar a un discurso y a prácticas sobre "la evaluación": "11 se requerirá de la intervención del psicólogo para "controlar" las metodologías utilizadas y proponer nuevas; su competencia es muy fácilmente identificable y la antigüedad de las tradiciones docimológicas es en general bastante conocida para que el recurso a tal función no represente problema alguno.

¿Pero esta metodología no se desarrolla de manera tan particular en el campo educativo que favorezca la formación de profesiones de evaluadores pedagogos, como parece organizarse actualmente al otro lado del Atlántico? Además, ¿el compromiso en vías de una evaluación formativa ${ }^{12}$ no lleva a interrogarse sobre la necesidad, más allá del llamado a una psicología funcional del aprendizaje, de cuestionar la psicología individual, y por lo tanto clínica?

La segunda imagen de la intervención de los psicólogos nos es proporcionada por los trabajos sobre la observación del comportamiento pedagógico de los maestros. El comportamiento verbal ha sido estudiado según algunos modelos inspirados en el interaccionismo de la escuela de psicología social americana; se nos presenta analizado por los defensores de un neo-conductismo que ya se ha concretizado en una cierta "pedagogía por objetivos". ${ }^{13}$ Pero incluso el comportamiento no-verbal ya es sujeto de investigación psicológica. ${ }^{14}$ Los investigadores prueban tablas de observación, minuciosamente elaboradas en situación real, luego cuidadosamente acabadas y probadas con video; los formadores utilizan estas herramientas para "concientizar" a los futuros maestros; después, los agentes de control institucional verifican, durante inspecciones de tiempo limitado, la adecuación de los comportamientos observados a las normas pedagógicas vigentes.

La psicología así utilizada ¿no es "puesta” al servicio de fines que le son ajenos? ¿No tiene nada que decir al respecto? ¿Por qué su silencio relativo?

España: Grijalbo. [N. de la T.]

II. Cf. Noizet y Caverni, I978: 232.

I2. Cf. Allal et al., I979.

I3. Cf. Besse, I977: II4-I40; Ketele, I980: 2I4 y Xuan y Chassain, I975: 192.

I4. Cf Landsheere y Delchambre, I979: 232. 


\section{3 Rol de la investigación fundamental}

La investigación psicológica con un objetivo fundamentalista no es aceptada espontáneamente, ni por las autoridades administrativas preocupadas con frecuencia por las repercusiones inmediatas en el plan de las aplicaciones posibles, ni por los mismos practicantes, escépticos ante la complejidad de las metodologías y temerosos frente a un estudio que los sitúa en algún lado, a pesar de las precauciones tomadas, como objetos de investigación, al mismo nivel que sus alumnos.

Por su parte, los investigadores no dejan de interrogarse sobre el impacto de sus trabajos; el balance no siempre lleva al entusiasmo. ${ }^{15}$ La situación francesa, desde este punto de vista, no deja de ser inquietante: comparativamente, países con población escolar menor, como Bélgica, Suiza o Canadá, disponen de servicios de investigación más consistentes, de medios notablemente más serios. Las universidades francesas deben reconocer las posibilidades adecuadas a la importancia de los trabajos a realizar para disponer de un conocimiento mejorado de las conductas desarrolladas en el campo escolar.

Esta investigación no puede ser garantizada más que por organismos que dispongan de la independencia necesaria, tanto frente a la administración escolar como a los practicantes de la enseñanza, y dotados de personal formado en la investigación de alto nivel por las universidades. La elección de los programas de investigación se beneficiaría al ser realizada por instancias plurales, donde los maestros, pero también los usuarios de esas formaciones (representantes de bachilleres, padres de alumnos, adultos en formación...) tendrían algo qué decir.

Sin duda, una investigación así, por los tiempos que requiere, por las precauciones metodológicas que son necesarias, no puede constituir el único modelo de referencia: ella es complementaria de otros enfoques, donde la polaridad “innovación” estaría más acentuada. Además, la investigación no puede dejar de preguntarse acerca de los efectos que induce tanto sobre el sistema educativo como sobre las condiciones mismas de su "producción de saber" y sus relaciones concretas con los educadores. Se trata, en resumidas cuentas, de buscar conciliar la voluntad de transformar la educación en función de objetivos definidos, de innovar pues, con la exigencia de una evolución conducida racionalmente.

I5. Cf. el coloquio sobre "L'apport des sciences fondamentales aux sciences de l'éducation ", Actes du $6^{\text {ème }}$ congrès international des Sciences de l'Éducation, Paris, Ed. Epi, 2 tomos, I974. 
Tales ambiciones suponen resueltas de antemano varias cuestiones importantes: evoquemos solamente la del estatus de la psicología de la educación, a la vez desde un punto de vista teórico y colocándola al nivel de su intervención concreta en el proceso de formación, inicial y continua, del personal docente. Al menos si no nos conformamos con evocar la existencia de una "psicopedagogía"...

\section{4 ¿Cuál psicopedagogía?}

Estudiando "la imagen de la psicopedagogía entre psicólogos y educadores", A. Leon (1967) y sus colaboradores proponen, al término de su análisis comparativo de dos revistas, la siguiente definición de la psicopedagogía, "disciplina a la vez teórica y práctica” (:603): “designa, en primer lugar, y es la acepción generalmente más moderada, una enseñanza que se apoya en el conocimiento de la psicología infantil y del adolescente. Pero tiene también como objeto la relación inversa y se propone estudiar las modificaciones psicológicas determinadas por una acción pedagógica" (:603). Las finalidades educativas que orientan la acción del profesor son susceptibles de ser "afectadas por los progresos de la psicología. Corresponde entonces a la psicopedagogía - es la tercera acepción consideradaaclarar las relaciones entre los fines de la educación y la actividad del psicólogo que opera en un medio escolar" (:603).

La relación presentada por una comisión ministerial encargada de estudiar "los problemas de psicología y de educación" (Institut National de Recherche Pédagogique [INRDP], I974: 28) nace por una tentativa de esclarecimiento de "la noción de psico-pedagogía", luego de haber subrayado que tal noción "se formó en la posteridad del pensamiento rousseano, para marcar la importancia de los resultados de la investigación psicológica en la definición de las actitudes pedagógicas y contribuir a la formación de los maestros" (:7). Pero no se trata de someter el estado de la práctica pedagógica al de los conocimientos psicológicos, porque "se llegaría a desvirtuar tanto a la psicología como a la pedagogía, confiriendo a la primera un carácter normativo que como ciencia no puede asumir y privando a la segunda del mismo carácter sin el cual no puede existir" (:8).

Los mismos autores se preguntan si la ambigüedad no se debería en parte al hecho de "que las cátedras de los profesores de escuela normal hagan explícitamente mención de la psicopedagogía mientras que son llamados en realidad a enseñar la filosofía de la educación y la antropología social como medios de la formación pedagógica" (INRDP, I974: 9). De hecho, es así como la "psicopeda- 
gogía" es enseñada actualmente, por algunos filósofos a quienes su formación inicial no permite sin duda prepararse para asumir los diferentes tipos de tareas asignadas por A. Léon a la psicopedagogía. ${ }^{16}$

En el marco de los Grupos de Ayuda Psico-pedagógicos (GAPP), ${ }^{17}$ la noción de psicopedagogía se encuentra en el título del "reeducador en psicopedagogía" (RPP): "de la especificidad misma del papel de psicopedagogo nace su complejidad. En efecto, el psico-pedagogo es un profesor, pero (...) de un tipo particular: debe cumplir su tarea de pedagogo sin perder de vista los objetivos terapéuticos de su acción" (Mery, I978: 22).

Así pues, la psicopedagogía puede ser percibida como una aplicación directa a la pedagogía de los conocimientos adquiridos en psicología infantil, o fijarse como objetivo el estudio de los fenómenos psicológicos que sobrevienen a partir de la educación escolar o bien proponerse una acción terapéutica en el campo escolar. G. Mialaret preferirá hablar de "pedago-psicología o de pedapsicología o de psicología de la educación" (Reuchlin, I97I) para describir "el conjunto de estudios de las conductas y procesos provocados o utilizados por la acción pedagógica".

¿Pero no se encuentra definida también la "pedagogía experimental” como "el estudio metódico de las reacciones de los alumnos a los procedimientos de enseñanza" (Simon, I924: I8) o incluso "el estudio de las interacciones entre el escolar y los medios de los cuales se vale la pedagogía para alcanzar sus fines" (Simon, 1972: 59)?

J. Leif (I960) propone distinguir claramente los dominios respectivos de la pedagogía y de la psicología infantil. Indica que la psicología puede hacer aportaciones específicas a la educación en los dominios siguientes:

- el comportamiento y la educación general del niño: psicología genética; psicología diferencial; psicoanálisis.

- los problemas de pedagogía general: la actividad del niño; el desarrollo de la inteligencia; los intereses; los tests; la distribución de los niños y su paso por los diferentes niveles de la escolaridad; los métodos activos; la orientación.

- los problemas de pedagogía especial: la lengua (Piaget); la enseñanza científica (Piaget, Wallon, Michaud); el cálculo (Piaget).

- la investigación pedagógica.

I6. Cf. en este punto: Coloquio de los profesores de filosofía de las E.N. IPN, Paris, I968, I2 p.; Fouilloud, I973; Ravoux, I974.

I7. Cf. la circular no. IV, 70-83, febrero 9, I970, BOEN no. 8, febrero I9, I970. 
Del lado de los psicólogos, las reservas con respecto a tales acepciones de la psicopedagogía no son pocas: se ve así descubierto el riesgo del "psicologismo" como "reducción de los fenómenos observados a un sólo factor, el factor psicológico, que termina por excluir la dimensión social e histórica y reduce al individuo particular a la causalidad de su evolución" (Lurcat, I974: I4). ¿La psicología no tiene también una función crítica frente a la institución: desde este punto de vista, la evolución de una cierta tendencia de la psicología social hacia el análisis institucional no es significativa de esta consideración de otras dimensiones?

Así, una verdadera incertidumbre parece reinar en las relaciones entre psicología y educación. Sin duda la educación, de manera mucho más evidente que para la psicología, echa raíces en inspiraciones filosóficas, en problemáticas ideológicas que se inscriben en una perspectiva histórica y social. Además, no nos parece posible comprender las posturas actuales de esas relaciones sin remontarse más a los orígenes mismos de la definición de los roles: con Alfred Binet y Ovide Decroly en particular.

\section{Binet, Decroly, Piaget: tres problemáticas}

No se puede decir que Binet, Decroly o Piaget hayan formulado proyectos relativos a la cuestión que nos preocupa aquí: la elección que hicimos -y que nos lleva a no considerar a $\mathrm{H}$. Wallon, por ejemplo ${ }^{18}$ - parece permitirnos delimitar qué problemas generales causan la articulación práctica entre psicología y educación y la elaboración de modelos funcionales que definen, de forma más teórica, esas mismas relaciones. Además, el orden de presentación que es adoptado, histórico y cronológico, deja de lado cualquier juicio a priori sobre el valor, mayor o menor, de cada una de estas posiciones.

\section{I Alfred Binet}

La unidad misma de la inspiración de la obra científica de Binet no es clara: se observa más bien como una vacilación, incluso una cierta inestabilidad en

I8. De quien se conoce sin embargo la parte activa que tomó en el mejoramiento de la educación. Cf Tran-Thong, I97I: I74. 
la definición de sus intereses. Si es considerado como uno de los psicólogos más creativos, pocos son los dominios que no haya explorado y en donde no haya manifestado sus opiniones e iniciado investigaciones llenas de lucidez. Ejerció su curiosidad en muy variados campos de la investigación, por lo que no debe sorprendernos ubicarlo también entre los pioneros de la investigación sistemática en psicología aplicada a la educación. De lo que se sabe menos es de la continuidad de estos trabajos y su creciente importancia durante los últimos años de su existencia.

Sin duda se trata, al menos para él, de "señalar las diferencias psicológicas individuales a fin de establecer experimentalmente una clasificación de los caracteres"19. Se reconoce aquí la ambición de constituir esta "psicología individual", que actualmente es denominada "psicología diferencial". En efecto, Binet se interesa más que nada en lo que él llama las diferencias de carácter que nosotros calificaremos más bien como aspectos intelectuales de la personalidad, o incluso como "estilos cognitivos", para compararlos con las investigaciones actuales.

La psicología individual así entendida es el estudio mismo de "las propiedades de los procesos psíquicos que varían de un individuo a otro" o incluso de las "variaciones que presenta el estado de las facultades según los individuos" (Binet \& Henri, I896: 4II). Éste seguirá siendo el objeto privilegiado de los trabajos de Binet.

La introducción de métodos científicos en pedagogía pronto se vuelve indispensable para él: una de las primeras tareas que cumplir consiste en "la determinación de las aptitudes de los niños (lo que) es la mayor preocupación de la enseñanza y de la educación. Se les debe instruir y dirigir hacia una profesión según sus aptitudes. La pedagogía debe tener como preámbulo un estudio de psicología individual" (Binet, I9IO: I I).

Para adquirir esos saberes, tanto del niño como del estudiante, Binet estima que la psicología, ya que se quiere científica, debe recurrir al método experimental, incluso si no es fácil mantenerse dentro de éste, toda vez que se tiene el proyecto de elaborar una "psicología individual"; se abstendrá de todas formas de lo no comprobable de la psicología "subjetiva". De alguna manera, en la clase se va a llevar a cabo una situación cuasi experimental, ya que el experimentador domina aquí, más o menos, las variables de la edad, del nivel de instrucción, de la influencia pedagógica... Para efectuar más fácilmente sus investigaciones psicológicas, ya dentro de las escuelas, Binet orientará sus trabajos cada vez más claramente hacia aplicaciones directamente pedagógicas.

I9. En La création littéraire, citado en Avanzini. I969: I8. 
Insistirá cada vez más en las consecuencias educativas de las investigaciones psicológicas, en particular luego de finalizada la escala métrica de la inteligencia y la posibilidad así obligada de construir una pedagogía individualizada sobre los reportes del conocimiento de las capacidades de cada uno. Recuerda entonces la necesidad de trabajar mejor con esta información acerca de las posibilidades individuales y preconiza diversas fórmulas de orientación escolar.

También el objetivo de sus investigaciones se desvía poco a poco. Tanto la psicología como la pedagogía son para él disciplinas objetivas; "les corresponde enseñarnos si los medios empleados están adaptados o no a esos fines (los de la actividad humana) o si presentan ventajas o inconvenientes que no se habían sospechado" (Binet, I9Iob: 87). No le parece suficiente "conocer" la psicología para poder enseñar eficazmente: cómo recopilar los saberes y hacerlos asimilar, tal es el objeto de la pedagogía, que debe constituirse en una pedagogía experimental, la cual "no es pura y simplemente una aplicación de las consecuencias de la psicología, sino una ciencia autónoma, que tiene sus propios métodos” (Binet, I9IOa, citado en Avanzini, I969: 233).

Sin embargo, si Binet percibe sobre todo que la "pedagogía científica debe tener una actitud reflexiva: bajo la doble perspectiva de la psicología individual que informa sobre sus aptitudes y normas que dan un objetivo a su educación, tiene como tarea proponer a cada niño, considerado particularmente, los procedimientos y los objetivos de trabajo que le convengan" (:234), él no define el objeto ni de la pedagogía experimental ni de la psicología pedagógica. "Lejos de caer en el olvido, esta imprecisión es más bien el signo de su incertidumbre".

Además, sobre la pregunta acerca de quién puede o debe administrar la escala métrica de la inteligencia, Binet no se decide; se limita a observaciones de orden metodológico acerca de las condiciones de aplicación, sin obligarse a una reflexión particular sobre una función que sería la del psicólogo. Más aún, si el objeto de su "pedagogía individual" es verdaderamente el estudio de las capacidades del niño, ¿cuál es entonces la especificidad de una psicología de la educación?

\section{2 Decroly}

Si la posteridad vio sobre todo en Binet a un psicólogo - ¡y de qué importancia!- para olvidar un poco lo que la investigación pedagógica le debía, se advierte casi lo contrario a propósito de Decroly; se conservan de él algunas creaciones pedagógicas notables, una institución, el método global de lectura, 
los centros de interés... se recuerda que fue un educador especialmente atento a la infancia, ordinaria e inadaptada: a menudo es situado en la frontera entre la psicología y la educación, ya que sería "el modelo mismo del psico-pedagogo" (Debesse, I968: 355).

Es esta particularidad de su obra la que le aseguraría la pertinencia y la credibilidad, pues por ello la acción pedagógica estaría “fundada en el conocimiento exacto del niño, de su naturaleza, de sus necesidades, de sus capacidades. Se basa en el estudio psicológico del niño". Es por eso que "la pedagogía y la psicología están unidas tan estrechamente como sea posible en la obra de Decroly" (Wallon, I952: I4). Pero Decroly psicólogo es desconocido, tal vez por el hecho de que esta parte de sus trabajos desaparece frente a la estatura del Decroly pedagogo: a propósito de esto, H. Wallon (I948) escribió que "hacerse sombra a sí mismo no está al alcance de todo el mundo" (:I).

Sin duda alguna, el enfoque decrolyano marca una etapa importante en la articulación de las relaciones entre psicología y educación: la originalidad de sus concepciones educativas se debe ante todo a su ambición de constituir una educación "científica" 20 . No permanece fuera de la acción pedagógica como lo estará Binet. Es en la confluencia cotidiana de sus investigaciones psicológicas y el mejoramiento de la educación en la École de l'Ermitage y en el Instituto para niños irregulares en donde sitúa sus esfuerzos.

\section{2. 1 La formación de Decroly}

Decroly extrajo de sus estudios en medicina y de sus primeros trabajos en neuropatología un gran interés por la investigación científica y una firme convicción sobre las ventajas notables del método experimental. Desde sus primeras publicaciones como médico psiquiatra manifiesta su interés por una acercamiento al mismo tiempo individualizante y apoyado en investigaciones objetivas.

La experiencia al lado de los niños inadaptados lo llevará rápidamente a crear una institución en la que se ve confrontado con los modelos pedagógicos entonces dominantes y de los cuales concluirá, luego de haberlos experimentado él mismo, su insuficiencia. Además no tardará en orientarse hacia las investigaciones de orden

20. Sobre el alcance y los límites de esta aspiración, cf. nuestro análisis sobre "Les doctrines d'inspiration rationaliste" (Besse, I98I: 75-95). 
psicológico para validar los procedimientos pedagógicos: la experimentación científica es indispensable para elaborar una pedagogía digna de ese nombre. En I906 escribirá por primera vez el proyecto de una "pedagogía experimental". ${ }^{21}$

En efecto, es indudable que adoptando una actitud científica es como se podrá determinar la selección óptima de las prácticas educativas, y afirma:

los métodos y los procedimientos así como el camino que hay que seguir deben ser sometidos no a lo que se denomina habitualmente la experiencia, que es casi siempre muy vaga (imprecisa) y demasiado personal, sino a la experimentación que, ella sola, puede poner a todo el mundo de acuerdo. (Decroly, I906: 547)

Sin embargo, la noción de "psicopedagogía" le es entonces extraña; más bien él habla de "pedología" y de "médico-pedagogía" para distinguir su proyecto. Si propone esta última formulación es para responder a los problemas particulares provocados por el tratamiento médico y pedagógico de la infancia "anormal": se trata entonces de apuntar a

la asociación del tratamiento médico con el pedagógico para la corrección de las anomalías psíquicas del niño. Esta asociación es indispensable en el caso en que, por razones diversas, la educación es imposible o difícil en la escuela ordinaria o bien en aquellos casos donde es necesario que se ejerza una vigilancia médica continua sin perjudicar la acción educativa sino, al contrario, dirigiéndola y corroborándola. (Decroly, I904: II9)

Aplicará la perspectiva y las ambiciones de la médico-pedagogía, inicialmente definida como la posibilidad de proponer para el niño anormal "un régimen mental y corporal individualizado, basado de manera constante en el conocimiento del estado psicológico y fisiológico". Los médicos escolares, formados en la experimentación durante sus estudios, tendrán que dirigir estas investigaciones y sus aplicaciones; esos "higienistas escolares piensan que deben poner atención hasta en el estado mental del niño y no solamente para prevenirlo contra posibles accidentes, sino para darle el régimen pedagógico apropiado a sus recursos intelectuales" (Decroly, I907: 23I).

En el pensamiento de Decroly, lo que al principio no es válido más que para la educación de los niños física o mentalmente discapacitados, va a apli-

2I. Decroly, en I906, escribía en su Psychologie du dessin: "Creemos - a nombre de todos los grandes intereses que dependen de la Escuela- que debemos llamar la atención de los hombres que se preocupan por el futuro de la enseñanza, sobre esta introducción de procedimientos de crítica científica en un dominio donde pareciera que éstos no debieran penetrar. No hay más que dar un paso para hacer entrar en la Escuela la herramienta más poderosa del progreso en todas las ramas de la actividad, a saber, la experimentación" (:546). 
carse después a la psicología y a la educación de los niños "ordinarios", porque los métodos educativos empleados al contacto con los "irregulares" (centros de interés, método "global" de lectura...) les funcionan tan bien ique sus resultados son comparados con aquéllos de los estudiantes "normales" de la escuela de a lado! Pero en todos los casos "el principio dominante debe ser siempre: adaptar la escuela al niño” (Decroly, I905: I7).

\section{2. 2 La necesidad, para la educación, de apoyarse en la psicología}

Para lograr "equilibrar el objetivo que se quiere alcanzar de acuerdo con las capacidades físicas y mentales del sujeto" (Decroly, I908: I24), conviene disponer de medios que permitan establecer la comprensión del psiquismo infantil, para enseguida evaluarlo eventualmente. Retomaremos el consejo de Rousseau y diremos que "por el momento, nosotros conducimos al niño, cuando es él quien debería conducirnos" (Decroly \& Degand, I907: 339). Si se quiere saber cómo puede el niño aprender a leer, se trata de ponerse a estudiar al niño, observar cómo percibe, cómo se interesa en los textos escritos... Decroly, en este caso, partió de investigaciones con niños sordos, luego con niños ciegos, a fin de apoyar sus observaciones sobre situaciones de experimentación "natural". Las experiencias en psicología experimental le permitirán concluir la superioridad de las modalidades de aprendizaje en las cuales el niño es puesto inmediatamente en contacto con frases significativas para su propia experiencia social.

La psicología infantil se acompaña de una psicología funcional y procede de igual manera en comparación con la psicopatología infantil; si cuando intervienen estas cuestiones "la psicología de los niños anormales es una ciencia en vías de constitución" (Decroly \& Demoor, I905: 3I7), también se puede esperar de ella "la elaboración de un método de tratamiento racional" así como el esclarecimiento del "mecanismo del pensamiento del niño normal".

La psicología que puede guiar a los educadores ya no es "esencialmente especulativa", ya que también estos últimos "se interesan activamente en las investigaciones positivas realizadas con el objetivo de definir exactamente los procesos múltiples del pensamiento en vías de evolución". Es por eso que para elaborar una educación racional "es necesario, y no hay otra salida, basarse en la psicología infantil, no del niño esquemático de los antiguos psicólogos, sino del ser vivo, variado, que evoluciona, sometido a las múltiples influencias de la herencia y del medio" (Decroly \& Degans, I906: 262). 


\section{2. 3 Una psicología diferencial}

El punto de referencia de la génesis del psiquismo no es suficiente sin embargo para permitir que el educador comprenda al niño. El estudio de las variaciones interindividuales es aquí indispensable y Decroly va a contribuir ampliamente a su establecimiento y a la difusión de sus aplicaciones.

Desde 1905 entró en contacto con Binet a propósito de la construcción de instrumentos propios para facilitar la evaluación de las capacidades de cada uno de los estudiantes. Será uno de los primeros en someter a una prueba la escala métrica de la inteligencia y procederá, a petición de Binet, a investigaciones comparativas acerca de la población escolar bruselense.

Los intereses de Decroly por la psicología genética se complementan poco a poco con investigaciones en psicometría, más susceptibles de aplicaciones pedagógicas $^{22}$. En efecto, el estudio de los tests le parece capaz de combinar el acercamiento experimental, la dimensión genética y la relación clínica, constituyendo una garantía contra el riesgo de subjetividad excesiva que implica la observación individual. Pone de manifiesto una intensa actividad para buscar convencer del valor de estos procedimientos. Con R. Buyse publica La pratique des tests mentaux [La práctica de los tests mentales] (I928) y elabora él mismo varias series de pruebas.

No olvida la importancia de la afectividad en el niño y su teoría de la afectividad le permite elaborar un inventario de comportamientos del alumno para emprender una observación continua. Luego, termina con un "cuestionario afectivo" destinado a facilitar el alcance de este objetivo. "Propusimos I 2 clases de caracteres tomando en cuenta tres factores: la actividad, la afectividad y la inteligencia (...). Retomando el tema (...) hicimos intervenir cuatro factores en lugar de tres y subdividimos varios de ellos. Sobre todo son factores:

- de orden físico (salud y fuerza)

- de orden nervioso (rapidez de reacción y dominio centrípeto o centrífugo de la actividad nerviosa)

- de orden afectivo (dominio del ego o exocéntricos)

- de orden intelectual (práctica o verbal, concreta o abstracta, global e intuitiva o analítica-sintética)". (Decroly, 1929)

La dificultad para la elaboración de una psicología diferencial se debe a motivos de diversa índole:

22. Sin embargo, sus Études de psychogenèse (1932: 346) indican mejor qué lugar ocupa en la historia de la psicología genética. 
la complejidad de la naturaleza humana es tal que es muy difícil delimitar los caracteres que permiten identificar con toda seguridad los tipos. La psicología diferencial e individual, en sus intentos por definir los caracteres de las razas, de los sexos, de los grupos profesionales o los de los individuos, se enfrenta a múltiples obstáculos. Entre éstos podemos señalar que las diferencias observadas no son más que diferencias promedio, de valor colectivo (...). Las combinaciones de efectos psíquicos son de tal manera numerosas que hay muy pocos tipos definidos y una multitud de tipos intermediarios. (Decroly, I929)

\section{2.4 ¿Una psicopedagogía?}

"El educador que no es observador será siempre un mal educador", apunta Decroly en su curso de Psicogénesis (I929). Además, es conveniente señalar que "el estudio del niño no debe aprenderse de memoria. Es necesario ponerse frente al niño mismo, tomar la actitud del jardinero, del médico" (I929). En su obra escrita como en su acción pedagógica en contacto con los educadores de la École de l'Ermitage, Decroly pone el acento en este conocimiento psicológico necesario para emprender la educación de los niños. Incorpora a los maestros de esta escuela a sus investigaciones, anima sus intercambios pedagógicos, les aporta informaciones e informes de investigación. La École de l'Ermitage dirigida por Decroly representa, desde este punto de vista, un modelo original de institución experimental donde la formación de los maestros, la investigación pedagógica y psicológica toman parte en un mismo movimiento. ¿Pero basta hablar entonces de una "psicopedagogía" para dar cuenta adecuadamente de tal procedimiento?

Sin duda Decroly se sitúa entre los hombres de ciencia que no se conforman con observar lo real y para quien es importante inscribirse en la dinámica de la transformación social. Eligió facilitar esas evoluciones insertándose en el terreno de la infancia y de la educación, y se preocupa en efecto por la necesidad de "cambiar la escuela". A fin de cuentas su procedimiento es el de un avance lento, racional y regular hacia esos objetivos. Decroly es pragmatista y tiene el cuidado de no adoptar ningún dogma, y mucho menos en pedagogía o en psicología. Preocupaciones concretas orientan su acción ${ }^{23}$.

23. Por mencionar un ejemplo: "Dos cuestiones deben regular el contenido de un año escolar: -¿qué es lo que el niño desea aprender como niño y qué debe saber como adulto?; ¿qué es lo que el niño en sus diferentes etapas de desarrollo puede aprender con provecho?" (Decroly \& 
¿Pero hemos encontrado en él respuestas seguras a nuestras preguntas sobre las relaciones entre psicología y educación? No parece así: Decroly teorizó poco sobre estos temas; pero es sobre todo el análisis de su obra lo que nos puede aclara este punto. En efecto, ¿no se observa algo como una ambigüedad frente a lo que convendría considerar como esencial del mensaje decroliano? El eclecticismo propio de las posiciones de Decroly lo ha llevado a intuiciones suficientemente pertinentes para que su obra motive a aplicaciones diferenciadas: no se podría emitir la hipótesis de que la escuela Decroly de Bruselas aplicaría más bien la polaridad más "racional", mientras que la de Saint-Mandé encontraría más la inspiración "vitalista"?

\section{3 Piaget}

La obra de Piaget responde a otras preocupaciones que no buscan aplicaciones directas ni psicológicas ni pedagógicas. Pero su obra ha sido frecuentemente usada para justificar tal o cual posición o innovación educativas ${ }^{24}$, y Piaget mismo, como presidente de la Oficina Internacional de Educación o en diversas publicaciones (I969, I97I), no dejó de intervenir en este debate, por lo que sin duda es importante estudiar lo que nuestro discurso puede tener de la empresa piagetiana.

Piaget, en su contribución de I935, tomó fuertemente partido por los “métodos nuevos" de educación, que se apoyarían en los descubrimientos de la psicología genética. Permanece sin duda como uno de los psicólogos más citados cuando los pedagogos experimentan la necesidad de justificar tal o cual acercamiento a los “trabajos psicológicos autorizados". Él mismo había indicado la posibilidad de utilizar, en pedagogía, los resultados de sus trabajos: "Siempre habíamos pensando que los materiales que nos fue posible reunir (...) así como las interpretaciones a las cuales estos hechos nos han conducido podrían dar lugar a una utilización pedagógica y en particular didáctica" (Piaget, I966; Cf. Piaget, I976).

¿Todavía conviene preguntarse si los adelantos de Piaget en el campo educativo proceden del mismo acercamiento que sus investigaciones en epistemo-

Buyse, I923: 53).

24. Cf. Porcher, Ferran, Mousset \& Simondin, I97I. "Actualmente, el conocimiento de la psicología genética se ha vuelto el preámbulo necesario para la acción pedagógica”. Cf. también Schwebel \& Raph, I976. 
logía genética? ¿No posee, en materia educativa, el discurso de un ciudadano, de un demócrata liberal interesado en la evolución general de los sistemas educativos que no efectúa trabajos metódicos, con su rigor habitual, relativos al objeto mismo de la educación? En efecto, se puede considerar que su estatus de científico le asegura el público necesario para expresarse sobre las cuestiones educativas, mientras que, por sí mismo, su obra científica ya no lo autoriza como a otros, a formular observaciones válidas. La autonomía del pedagógico introduce aquí una ruptura, y limita otro tanto el alcance de las opiniones de Piaget sobre este problema.

Así, una vez que llegamos al término de la confrontación esbozada entre estos tres psicólogos, aparece todavía más claramente la complejidad de nuestra empresa: Binet se esfuerza por mejorar el conocimiento que nosotros podemos tener del estudiante y preconiza un estudio sistemático de los efectos de la pedagogía; Decroly interviene en el terreno mismo de la educación y utiliza los conocimientos psicológicos disponibles; Piaget permanece al margen y se ocupa esencialmente del sujeto epistémico. ¿Dónde situar entonces la psicología de la educación?

\section{III ¿Una psicología de la educación?}

Los saberes elaborados por la psicología no pueden dejar de ser recuperados por los pedagogos para emplearlos en su propio quehacer. Pero la manera en la que son utilizados esos conocimientos no puede ser indiferente a los que producen ese saber ${ }^{25}$. Además, el sistema educativo requiere de psicólogos para promover cambios en las actitudes y los comportamientos de los maestros o para intervenir directamente en la detección de las dificultades de los estudiantes y su orientación; la investigación pedagógica integra dimensiones específicas a la investigación psicológica, incluso si ella recluta psicólogos sobre todo con fines de control evaluativo.

La articulación, concreta y teórica a la vez, entre los aportes y los objetivos específicos de la psicología y de la educación o de la formación no se produce espontáneamente; de los diferentes modelos que acabamos de evocar ninguno

25. Cf. por ejemplo los trabajos por "el efecto Pigmalión”: Rosenthal \& Jacobson, I97I; Carlier \& Gottesdiener, I975. 
parece totalmente satisfactorio. Y sin duda no bastaría limitarse a un eclecticismo pragmático, dispuesto a justificar todo, y que llegara a constituir como "psicología de la educación" una serie yuxtapuesta de temas de investigación, listas de aplicaciones posibles de trabajos psicológicos en pedagogía y a tratar de modo contractual a los psicólogos según modalidades confusas, dando a veces los pedagogos la impresión de elegir su psicología ${ }^{26}$.

La psicología, general u orientada más específicamente a la educación, no puede evitar conservar una actitud distanciada con relación a la intención de instruir o de educar; no está orientada por un proyecto educativo, por objetivos didácticos y no llega pues a apoyar tal o cual disposición pedagógica (aunque Decroly, y por momentos Piaget, como lo hemos visto, se atrevieron a confirmar un procedimiento pedagógico por su autoridad científica): el psicólogo, confrontado con la acción pedagógica, está al mismo tiempo con los educadores, para comprenderlos, recoger desde el interior sus preocupaciones y sus ambiciones, pero también está en el exterior de la pedagogía, dentro y fuera. Posición difícil, no exenta de ambigüedades latentes, pero única posición defendible si el psicólogo quiere poder decir algo distinto de lo que está contenido en el discurso pedagógico.

26. Incluso algunas veces los psicólogos van a proponer/imponer un modelo total; ¿no es acaso la tendencia de B.F. Skinner en Par delà la liberté et la dignité (1972)?. 


\section{Lista de referencias}

Allal, L., Cardinet, J. \& Perrenoud, Ph. (1979). L'évaluation formative dans un enseignement différencié. Suiza: Peter Lang.

Andrey, B. \& Le Men, J. (I974). La psychologie à l'école. Paris: Puf.

Avanzini, G. (1969). Alfred Binet et la pédagogie scientifique. Paris: Vrin.

(I975). Immobilisme et novation dans l'éducation scolaire. Toulouse: Privat.

Besse, J. M. (I977). Vers une pédagogie par objectifs? Bull. Soc. Binet-Simon, III (556), pp. II4-I4O.

(I98I). Les doctrines d'inspiration rationaliste. En G. Avanzini (Coord.), Histoire de la pédagogie du 17ème siècle à nos jours, pp. 75-95. Toulouse: Privat.

Binet, A. (I9Iоa). Avant-propos. Année psychologique, t. I6. 6. Citado en Avanzini, G. (I969), Alfred Binet et la pédagogie scientifique, p. 233. Paris: Vrin.

(I9Iob, febrero). La neutralité scolaire. Bulletin Société Libre pour l'étude psychologique de l'enfant, 60.

(I9IOc). Les idées modernes sur les enfants. Paris: Flammarion.

Binet, A. \& Henri. (I896). La psychologie individuelle. Année psychologique, t. 2. Buyse, R. (I935). L'expérimentation pédagogique. Bruselas: Lamertin.

Carlier, M. \& Gottesdiener, H. (I975). Effet de l'expérimentateur, effet du mâ̂tre, réalité ou illusion? Enfance no. 2, pp. 219-24I.

Celma, J. (I97I). Journal d'un éducastreur. Paris : Champ Libre.

Debesse, M. (I968, diciembre). Psychologie de l'éducation. Bulletin de Psychologie, 274, XXII, 5-6.

Decroly, J. O. (I904). La médico-pédagogie. Ann. Soc. Méd. Gand., 84. et médicaux.

(I905). Organisation des écoles et institutions pour arriérés pédagogiques (I906). Psychologie du dessin. 1'École Nationale, 5(I8).

(1907). La psychologie, la pathologie et le traitement des enfants anormaux. Bull. Soc. Méd. Ment. de Belgique. (1908). Principes généraux relatifs au traitement des enfants irréguliers. (I929). Manuscrito inédito. Reproducido en "Le Dr. Decroly et l'éducation", (I972), Documents pédagogiques, Fasc. I3-I4. Bruselas: École Decroly. (I932). Études de psychogenèse. Bruselas: Lamertin. 
Decroly, J. O. \& Buyse, R. (I923). Les applications américaines de la psychologie. Bruselas: Lamertine. (1928). La pratique des tests mentaux. Paris: Alcan.

Decroly, J. O. \& Degans. (I906). Quelques considérations sur la psychologie et la pédagogie de la lecture. Revue Scientifique.

(I907). Contribution à la pédagogie de la lecture et de l'écriture. Arch. de Psych.

Decroly, J. O. \& Demoor, J. (I905). Revue de Pédagogie des Anormaux. Année Psych,

Fouilloud, C. (I973, octubre). Le professeur de psychopédagogie dans les E.N. Revue del SNPEN, 37.

Gilly, M. (I980). Maître-élève, Rôles institutionnels et représentations. Paris: PUf.

Gilly, M. (I98I). Psychologie de 1’Éducation : discipline appliquée ou fondamentale?, Bulletin de Psychologie, Xxxv (I-5), 353, pp. 3-9.

Institut National de Recherche Pédagogique [INRDP]. (I974). L'enseignement de la psychologie dans la formation des maîtres. SEVPEN, 2062.

Ketele, J. M. (I980). Observer pour éduquer. Suiza: P. Lang.

Landsheere, G. de \& Delchambre, A. (I979). Les comportements non-verbaux de l'enseignant. Comment les maîtres enseignent, II. Paris: Nathan.

Legrand, L. (I96I). Psychologie appliquée à l'éducation intellectuelle. Francia: Delachaux y Niestlé

(I968, septiembre I2). À propos de l'An 1 de la recherche pédagogique, Le Monde.

(I976, marzo). L'évolution des CES expérimentaux. Les Amis de Sèvres, I, pp. II-27.

(I977). Pour une politique démocratique de l'éducation. Paris: Puf.

Leif, J. (1960). Psychologie de l'enfant et recherche pédagogique. Encyclopédie pratique de l'éducation en France. París: IPN-SEDE.

Leon, A. (1967). L'image de la psychopédagogie chez des psychologues et des éducateurs : étude comparée de deux revues. Bulletin de Psychologie, xx (257), IO-I5, pp. 596-604.

Lurcat, L. (1974). Psychologisme et psychologie. 1'Éducation, 3I.I.

Maisonneuve, J. (I975). Présentation du Laboratoire de Psychologie Sociale et Sciences de 1'Éducation, Bulletin de Psychologie, XxviII(9-I2), 3I6, pp. 469470.

Marc, P. (1977). Les psychologues dans l'institution scolaire. Paris: Centurion.

Mery, J. (I978). Pédagogie curative scolaire et psychanalyse. Paris: ESF. 
Noizet, G. \& Caverni, J.P. (I978). Psychologie de l'évaluation scolaire. Paris: Puf.

Piaget, J. (I966). Prefacio. En H. Aebli, Didactique psychologique, Francia: Delachaux y Niestlé.

(1969). Psychologie et Pédagogie. Paris: Denoël.

(I972). Où va l'éducation. Paris: Denoël.

(I976), octubre-diciembre). Une heure après Piaget (à propos de l'enseignement des mathématiques). Revue Française de Pédagogie, no. 37, pp. 5-I2.

Piéron, H. (1957). Vocabulaire de la psychologie. Paris: PUF.

Porcher, L., Ferran, P., Mousset, R., \& Simondin, M. (I97I). Pour comprendre et pratiquer les activités d'éveil. Paris: Bourrelier.

Ravoux J.P. (I974, febrero). Sur la nature, le sens et la nécessité de la tâche du psychopédagogue dans les Écoles Normales. Revue du SNPEN, 4I.

Reuchlin M. (I97I). La psychologie de l'éducation. En Traité de psychologie appliquée, tomo I, capítulo II, pp. 55-I03. Paris: PUf.

Roller, S. (I968, julio-diciembre). L'aspect institutionnalisé de la recherche en Europe. Les Sciences de l'Éducation, 3-4, pp. 5I-57.

Rosenthal, R. \& Jacobson, L. (I97I). Pygmalion à l'école. Paris: Casterman.

Schwebel, M. \& Raph, J. (I976). Piaget à l'école. Paris: Denoël-Gonthier.

Selvini Palazzoli, M. et al. (I980). Le magicien sans magie. Paris: ESF.

Simon, J. (I972). La pédagogie expérimentale. Toulouse: Privat.

Simon, T. (I924). La pédagogie expérimentale. Paris: A. Colin.

Skinner, B.F. (I972). Par delà la liberté et la dignité? Francia: Laffont.

Tran-Thong. (I97I). La pensée pédagogique d'Henri Wallon. Paris: PUF.

Wallon, H. (1948). Prefacio. En J.E. Segers, La psychologie de l'enfant normal et anormal d'après le Dr Decroly. Bruselas: R. Stoops.

(I952). Hommage au Dr Decroly. Bruselas: Ministère de l'instruction publique.

Xuan, Le \& Chassain, J. C. (1975). Analyse comportementale. Paris: Nathan. 